\title{
Operator mixing in fermionic CFTs in noninteger dimensions
}

\author{
Yao $\mathrm{Ji}^{1, *}$ and Alexander N. Manashov ${ }^{2,1,3, \dagger}$ \\ ${ }^{1}$ Institut für Theoretische Physik, Universität Regensburg, D-93040 Regensburg, Germany \\ ${ }^{2}$ Institut für Theoretische Physik, Universität Hamburg, D-22761 Hamburg, Germany \\ ${ }^{3}$ St. Petersburg Department of Steklov Mathematical Institute, 191023 St. Petersburg, Russia
}

(Received 6 September 2018; published 1 November 2018)

\begin{abstract}
We consider the renormalization of four-fermion operators in the critical QED and $S U\left(N_{c}\right)$ version of the Gross-Neveu-Yukawa model in noninteger dimensions. Since the number of mixing operators is infinite, the diagonalization of an anomalous dimension matrix becomes a nontrivial problem. At leading order, the construction of eigenoperators is equivalent to solving certain three-term recurrence relations. We find analytic solutions of these recurrence relations that allow us to determine the spectrum of anomalous dimensions and study their properties.
\end{abstract}

DOI: 10.1103/PhysRevD.98.105001

\section{INTRODUCTION}

Quantum field theories (QFTs) in noninteger dimensions $d<4$ were introduced as a tool to calculate critical exponents in three-dimensional systems at a phase transition point [1]. As a rule, QFTs in $d=4-2 \epsilon$ possess nontrivial critical points with coupling constants being of order $\epsilon$. It allows one to calculate critical dimensions as power series in $\epsilon$ and extrapolate results to $\epsilon=1 / 2$. The current state of the art $\epsilon$-expansion technique and the corresponding references can be found, e.g., in Refs. [2,3].

It is clear, however, that QFTs in noninteger dimensions are not full fledged quantum field models — no real physical system is described by these QFTs. Thus they are not obliged to comply with expectations based on physical principles. It was shown in Ref. [4], in the example of $\varphi^{4}$ theory, that such models are necessarily nonunitary. In the $\varphi^{4}$ model, the lowest state with a negative norm is associated with an operator of rather high dimension $(\Delta=15)$, and the first complex anomalous dimensions appear for operators of dimension $\Delta=23$. Therefore, one may hope that the effect of these states on, e.g., the operator product expansion (OPE), could be neglected. In the fermionic models, however, the negative norm operators have a rather low, $\Delta=6$, canonical dimension [5] and can hardly be ignored.

Physical observables in conformal field theories (CFTs) are correlation functions of local operators. One is interested, in particular, in their behavior under scale and conformal

\footnotetext{
yao.ji@ur.de

†alexander.manashov@desy.de
}

Published by the American Physical Society under the terms of the Creative Commons Attribution 4.0 International license. Further distribution of this work must maintain attribution to the author(s) and the published article's title, journal citation, and DOI. Funded by SCOAP ${ }^{3}$. transformations. Therefore the basis of operators which transform in a proper way under scale and conformal transformations plays a distinguished role. In perturbation theory, such a basis is constructed by diagonalization of the anomalous dimension matrices. Since only operators of the same canonical dimension mix under renormalization, such a matrix has a finite size in scalar field theories. In a fermionic QFT, the situation is quite different - the number of mixing operators is, in most cases, infinite. The simplest example of this kind is given by the four fermion operators,

$$
\mathcal{O}_{n}=\frac{1}{n !}\left(\bar{q} \Gamma_{n}^{\mu_{1} \ldots \mu_{n}} q\right)\left(\bar{q} \Gamma_{\mu_{1} \ldots \mu_{n}}^{n} q\right),
$$

where $n=0,1, \ldots$ and $\Gamma_{\mu_{1} \ldots \mu_{n}}^{n}$ is the antisymmetrized product of the $d$-dimensional $\gamma$-matrices. All these operators have canonical dimension $\Delta=6$ and mix under renormalization. Customarily, the operators with $n \leq 4$ are called physical operators, and all others, since they vanish in $d=4$, evanescent ones.

In the QCD context, four-fermion operators arise in the description of nonleptonic weak decays of hadrons. Their renormalization was studied in [6-8]. It was shown in [7] that the mixing between evanescent operators and the physical ones can be avoided by an appropriate modification of the subtraction scheme.

Here we are interested in a different challengeconstructing operators which have certain scaling dimensions at a critical point. Since the size of the mixing matrix for the operators (1) is infinite, it is far from obvious that it can be done in all situations. Solving the eigenvalue problem, one has to impose certain requirements (quantization conditions) on the solutions. Since we are interested in determining the scaling properties of the correlators of operators (1), in particular the simplest one $\left\langle\mathcal{O}_{n}(x) \mathcal{O}_{m}(0)\right\rangle$, 
it is reasonable to require the correlation functions between two eigenoperators to be finite, i.e., for $\mathcal{O}_{\Delta}=$ $\sum_{n=0}^{\infty} c_{n}(\Delta) \mathcal{O}_{n}$,

$$
\left\langle\mathcal{O}_{\Delta}(x) \mathcal{O}_{\Delta}(0)\right\rangle<\infty .
$$

This condition is always fulfilled if the mixing matrix has a finite size, as in the case of scalar field theories, but leads to nontrivial "quantization" conditions for infinite matrices.

In this work, we consider renormalization of fourfermion operators in two theories: the critical QED and $S U\left(N_{c}\right)$ Gross-Neveu-Yukawa (GNY) models. The first model (QED) was used as an example by Dugan and Grinstein in their analysis [7]. In both cases, the spectral problem is equivalent to solving certain three-term recurrence relations. We present analytic solutions to these recurrence relations and discuss the possibility to satisfy the condition (2).

The paper is organized as follows: In Sec. II, we present the solution to the one-loop mixing problem for the operators (1) in QED. The operator mixing in the GNY model is discussed in Sec. III. We introduce an extended GNY model in Sec. IV and study the renormalization of four-fermion operators in this model.

\section{CRITICAL QED}

In $d=4-2 \epsilon$ dimensions, QED with $N_{f}$ fermions has an infrared stable critical point at $a=a_{*}\left(a=e^{2} / 4 \pi^{2}\right)$

$$
a_{*}=3 \epsilon / N_{f}+O(\epsilon) .
$$

At the critical coupling, the theory (in the Landau gauge) is scale invariant. ${ }^{1}$

Renormalization of four-fermion operators (1) in QED was studied in [7]. In order to avoid unnecessary complications, it is convenient to assume that the fermion and antifermion fields have different flavors. At the critical point the renormalized operators $[\mathcal{O}]_{n}$ satisfy the renormalization group equation,

$$
\left(\delta_{n m} M \partial_{M}+\gamma_{n m}\right)[\mathcal{O}]_{m}=0,
$$

where $M$ is the renormalization scale and $\gamma_{n m}$ is the anomalous dimension matrix. At one loop, the matrix $\gamma$ takes the following form [7]:

$$
\begin{aligned}
\gamma_{n m}= & \frac{a_{*}}{2}\left[(n+2)(n+1) \delta_{n+2, m}-2(n-1)(n-3) \delta_{n, m}\right. \\
& \left.+(n-5)(n-6) \delta_{n-2, m}\right] .
\end{aligned}
$$

\footnotetext{
${ }^{1}$ The model can also be analyzed with the $1 / N_{f}$ expansion technique; see Ref. [9] for a review and references. For a discussion of the three-dimensional model $\left(\mathrm{QED}_{3}\right)$ and its critical properties; see, e.g., [10-12].
}

In order to construct an operator with a certain scaling dimension, $\mathcal{O}_{\gamma}=\sum_{n} c_{n} \mathcal{O}_{n}$, one has to find the left eigenvectors of the matrix $\gamma$,

$$
\sum_{n} c_{n} \gamma_{n m}=\gamma c_{m} \equiv \frac{1}{2} a_{*} \bar{\gamma} c_{m}
$$

Since there is no mixing between the operators $\mathcal{O}_{n}$ with odd and even index $n$, each set can be analyzed separately. The analysis in both cases goes along the same line, and we therefore consider odd $n$ only.

The transpose matrix $\gamma^{T}$ (from now on the indices $n, m$ take odd values) has a three-diagonal form

$$
\boldsymbol{\gamma}^{T}=\frac{a_{*}}{2}\left(\begin{array}{ccccccc}
a_{1} & b_{3} & 0 & 0 & 0 & 0 & \ldots \\
d_{1} & a_{3} & 0 & 0 & 0 & 0 & \ldots \\
0 & d_{3} & a_{5} & b_{7} & 0 & 0 & \ldots \\
0 & 0 & d_{5} & a_{7} & b_{9} & 0 & \ldots \\
\ldots & \ldots & \ldots & \ldots & \ldots & \ldots & \ldots
\end{array}\right),
$$

where $a_{n}=-2(n-1)(n-3), b_{n}=(n-5)(n-6), d_{n}=$ $(n+2)(n+1)$ and we take into account that $b_{5}=0$. The two-by-two block in the upper-left corner describes mixing between the physical operators, $\mathcal{O}_{1}$ and $\mathcal{O}_{3}$. The corresponding eigenvalues are $\bar{\gamma}_{ \pm}= \pm 6$. The eigenvectors corresponding to these eigenvalues take the form

$$
c_{n}^{+}=1, \quad c_{n}^{-}=(n-2), \quad n \text { is odd },
$$

and the two operators $\mathcal{O}^{ \pm}$are

$$
\mathcal{O}^{+}=\sum_{n \in \mathbb{N}_{-}} \mathcal{O}_{n}, \quad \mathcal{O}^{-}=\sum_{n \in \mathbb{N}_{-}}(n-2) \mathcal{O}_{n},
$$

where sums go over odd integers.

All other eigenvectors of the matrix $\gamma$ have the form $\vec{c}=\left(0,0, c_{5}, c_{7}, \ldots\right)$. Indeed, the subspace spanned by these vectors is an invariant subspace of the matrix $\boldsymbol{\gamma}$. Looking for solutions in the form $c_{2 k+5}=s_{k}(2 k+5) ! /(2 k)$ ! with $k=0,1,2, \ldots$, one gets the following recurrence relation,

$$
C_{k} s_{k-1}-\left(A_{k}+C_{k}\right) s_{k}+A_{k} s_{k+1}=\frac{1}{4}(\bar{\gamma}-26) s_{k},
$$

where $C_{k}=k(k-1 / 2) \quad$ and $\quad A_{k}=(k+3)(k+7 / 2)$. The above equation is nothing but the recurrence relation for the continuous dual Hahn polynomials [13,14]. Its solutions take the form

$$
s_{k}(\nu)={ }_{3} F_{2}\left(\begin{array}{c|c}
-k, 3+i \nu, 3-i \nu & 1 \\
3,7 / 2 & 1
\end{array}\right),
$$

where $\nu$ is given by $\nu^{2}=(-\bar{\gamma}-10) / 4$. For large $k$, the coefficients $s_{k}(\nu)$ have a powerlike behavior

$$
s_{k}(\nu) \underset{k \rightarrow \infty}{\sim} r(\nu)(2 k)^{i \nu-3}+r(-\nu)(2 k)^{-i \nu-3}+\ldots,
$$


where $r(\nu)=15 \cdot 2^{i \nu} / \Gamma(3+i \nu)$. These functions form a complete orthonormal system on $L^{2}\left(\mathbb{R}_{+}\right)[13,14]$

$$
\int_{0}^{\infty} d \nu \mu(\nu) s_{k}(\nu) s_{n}(\nu)=\delta_{n k} \frac{(2 k) !}{(2 k+5) !},
$$

where

$$
\mu(\nu)=\frac{1}{225} \frac{\nu\left(1+\nu^{2}\right)\left(4+\nu^{2}\right)}{\sinh \pi \nu} .
$$

In order to fix the allowed values of $\nu$, let us consider the correlator of two eigenoperators:

$$
\mathcal{O}_{\nu}(x)=\sum_{k \geq 0} s_{k}(\nu) \mathcal{O}_{2 k+5}(x) .
$$

Note that the sum involves evanescent operators only. The operators $\mathcal{O}_{\nu}$, as follows from Eq. (11), are even functions of $\nu, \mathcal{O}_{\nu}=\mathcal{O}_{-\nu}$.

The leading order correlator of two basic operators (all fields have different flavors) was calculated in [5]

$$
\left\langle\mathcal{O}_{n}(x) \mathcal{O}_{m}(0)\right\rangle=\delta_{m n} \frac{24 \omega(n)}{\pi^{8}|x|^{12-8 \epsilon}},
$$

where

$$
\omega(n)=\left\{\begin{array}{ll}
1 / n !(4-n) ! & n \leq 4 \\
2 \epsilon(-1)^{n}(n-5) ! / n ! & n \geq 5
\end{array} .\right.
$$

Note that for the evanescent operators, $n \geq 5$, the weight factor $\omega(n)$ is proportional to $\epsilon$ and sign changing.

Then for the eigenoperators (15), one obtains

$$
\left\langle\mathcal{O}_{\nu}(x) \mathcal{O}_{\nu^{\prime}}(0)\right\rangle=|x|^{-12+8 \epsilon} R\left(\nu, \nu^{\prime}\right),
$$

where the residue $R\left(\nu, \nu^{\prime}\right)$ is given by the sum

$$
R\left(\nu, \nu^{\prime}\right) \sim-\epsilon \sum_{k} \frac{(2 k+5) !}{(2 k) !} s_{k}(\nu) s_{k}\left(\nu^{\prime}\right) .
$$

For large $k$, the summand decays as $k^{-1 \pm i \nu \pm i \nu^{\prime}}$ and $k^{-1 \pm i \nu \mp i \nu^{\prime}}$. Thus, the sum diverges if $\nu$ has a nonzero imaginary part. For real $\nu$, the correlator (18) can be understood in the sense of distributions. Assuming that $\nu, \nu^{\prime} \geq 0$ and taking into account Eq. (13), we get

$$
\sum_{k} \frac{(2 k+5) !}{(2 k) !} s_{k}(\nu) s_{k}\left(\nu^{\prime}\right)=\mu^{-1}(\nu) \delta\left(\nu-\nu^{\prime}\right) .
$$

For the correlator, it results in

$$
\left\langle\mathcal{O}_{\nu}(x) \mathcal{O}_{\nu^{\prime}}(0)\right\rangle=-\frac{48 \epsilon}{|x|^{2 \Delta_{\nu}}} \mu^{-1}(\nu) \delta\left(\nu-\nu^{\prime}\right),
$$

where we have included the one-loop correction to the operator dimension $|x|^{-12+8 \epsilon} \rightarrow|x|^{-2 \Delta_{\nu}}$ with

$$
\Delta_{\nu}=6-4 \epsilon-2 a_{*}\left(\frac{5}{2}+\nu^{2}\right) .
$$

Note that the anomalous dimensions of evanescent operators are negative.

The relation inverse to Eq. (15) reads

$$
\mathcal{O}_{2 k+5}(x)=\int_{0}^{\infty} d \nu \mu(\nu) s_{k}(\nu) \mathcal{O}_{\nu}(x) .
$$

It results in the following expression for the correlator of two (one-loop renormalized) evanescent operators (1)

$$
\left\langle\mathcal{O}_{n}(x) \mathcal{O}_{n^{\prime}}(0)\right\rangle=-48 \epsilon \int_{0}^{\infty} d \nu \mu(\nu) \frac{s_{k}(\nu) s_{k^{\prime}}(\nu)}{|x|^{2 \Delta_{\nu}}} .
$$

where $n=2 k+5, n^{\prime}=2 k^{\prime}+5$.

Coming back to the physical operators $\mathcal{O}_{ \pm}$, we note that these operators contain an infinite tail of evanescent operators; see Eq. (9). The contribution of the evanescent operators to the correlators, $\left\langle\mathcal{O}_{ \pm}(x) \mathcal{O}_{ \pm}(0)\right\rangle$ is of order $\epsilon$ and, strictly speaking, beyond our accuracy. Nevertheless, we stress that the corresponding sum converges.

Since the operators $\mathcal{O}_{ \pm}$have different scaling dimensions, their correlator has to vanish. One can easily check using (9) that $\left\langle\mathcal{O}_{+}(x) \mathcal{O}_{-}(0)\right\rangle \sim O(\epsilon)$ as it should be. It can also be easily checked that the correlator of an evanescent operator with the physical one is of order $\epsilon$ as well, $\left\langle\mathcal{O}_{\nu}(x) \mathcal{O}_{ \pm}(0)\right\rangle=O(\epsilon)$.

\section{OPERATOR MIXING IN THE GROSS-NEVEU-YUKAWA MODEL}

In this section, we briefly consider the specifics of operator mixing in the GNY model [15]. The one-loop anomalous dimension matrix for the four-quark operators (1) has been calculated in Ref. [5]. It has the following structure: the operator $\mathcal{O}_{n=0}$ is renormalized multiplicatively, and the anomalous dimensions matrix for the operators $\mathcal{O}_{n}, n \geq 1$ has a block-diagonal form,

$$
\boldsymbol{\gamma}=\operatorname{diag}\left(A_{1}, A_{3}, A_{5}, \ldots\right)
$$

where each block $A_{k}$, with $k$ being odd, describes the mixing between the operators, $\mathcal{O}_{k}$ and $\mathcal{O}_{k+1}$. The blocks $A_{k}$ depend nontrivially on $k$ but all have the same eigenvalues. Thus at the one-loop level, there are only two different anomalous dimensions, $\gamma_{ \pm}$, which correspond to two different eigenvalues of the blocks $A_{k}$. The anomalous dimension of the operator $\mathcal{O}_{n=0}$ coincides with $\gamma_{+}$.

Surprisingly enough, the matrix $\boldsymbol{\gamma}$ preserves this form at the two-loop order as well. We obtain the following expression for the block $A_{k}$ 


$$
\begin{aligned}
A_{k}= & 2 u_{*}\left(1-u_{*} \frac{n_{f}+12}{4}\right)\left(\begin{array}{cc}
k-1 & -1 \\
-(k+1)(4-k) & 2-k
\end{array}\right) \\
& -\frac{1}{2} u_{*}^{2}\left(\begin{array}{cc}
19 & 0 \\
4(k+1)\left(4-n_{f}\right) & 4 n_{f}+3
\end{array}\right),
\end{aligned}
$$

where $n_{f}=N_{f} \times \operatorname{tr} \mathbb{1}$ and the critical value $u_{*}$ for the GNY model reads $[15,16]$

$$
u_{*}=\frac{2 \epsilon}{n_{f}+6}\left(1+\frac{12 \epsilon}{n_{f}+6}\right)+O\left(\epsilon^{3}\right) .
$$

The eigenvalues of the blocks $A_{k}$ do not depend on $k$,

$$
\begin{aligned}
& \gamma^{+}=6 u_{*}\left(1-u_{*} \frac{7 n_{f}+36}{12}\right), \\
& \gamma^{-}=-4 u_{*}\left(1-u_{*} \frac{2 n_{f}+5}{8}\right),
\end{aligned}
$$

and the anomalous dimension of the operator $\mathcal{O}_{n=0}$ is still equal to $\gamma^{+}$.

The explanation for such degeneracy of the anomalous dimensions is the following: let us consider two sets of operators,

$$
\begin{aligned}
\mathcal{O}_{n} & =\left(\bar{\psi}_{1} \Gamma_{n} \psi_{2}\right)\left(\bar{\psi}_{3} \Gamma_{n} \psi_{4}\right), \\
\mathcal{O}_{n}^{\prime} & =\left(\bar{\psi}_{1} \Gamma_{n} \psi_{4}\right)\left(\bar{\psi}_{3} \Gamma_{n} \psi_{2}\right) .
\end{aligned}
$$

The operator $\mathcal{O}_{n}$ and $\mathcal{O}_{n}^{\prime}$ obey exactly the same RG equation. At the same time, they are related to each other by Fierz transformation (A4),

$$
\mathcal{O}_{n}^{\prime}=\sum_{m} \Omega_{n m}(d) \mathcal{O}_{m}
$$

Going over to the renormalized operators, one gets

$$
\left[\mathcal{O}^{\prime}\right]_{n}=\sum_{m} \tilde{\Omega}_{n m}(d)[\mathcal{O}]_{m},
$$

where $[\mathcal{O}]_{n}=Z_{n m} \mathcal{O}_{m}\left(\left[\mathcal{O}^{\prime}\right]_{n}=Z_{n m} \mathcal{O}_{m}^{\prime}\right)$ and

$$
\tilde{\Omega}(d)=Z \Omega(d) Z^{-1} .
$$

The matrix $\tilde{\Omega}$ is a finite matrix (has no $\epsilon$ poles) of infinite size which depends on $\epsilon$ and the coupling constants. Taking the derivative $M \partial_{M}$ on both sides of Eq. (31), one finds that at the critical point, the anomalous dimension matrix $\gamma$ commutes with $\tilde{\Omega}$,

$$
\gamma \tilde{\Omega}=\tilde{\Omega} \gamma .
$$

Then, provided that the matrix $\boldsymbol{\gamma}$ has a block diagonal form (25), it follows that the matrix

$$
\tilde{\Omega}^{(k m)}=\left(\begin{array}{cc}
\tilde{\Omega}_{k, m} & \tilde{\Omega}_{k, m+1} \\
\tilde{\Omega}_{k+1, m} & \tilde{\Omega}_{k+1, m+1}
\end{array}\right)
$$

intertwines the blocks $A_{k}$ and $A_{m}$,

$$
A_{k} \tilde{\Omega}^{(k m)}=\tilde{\Omega}^{(k m)} A_{m} .
$$

Hence, they have the same eigenvalues, as $\tilde{\boldsymbol{\Omega}}^{(\mathrm{km})}$ is a convertible matrix.

In a similar manner, one can easily show that the vector $\vec{c}_{k}=\left(\tilde{\Omega}_{0, k}, \tilde{\Omega}_{0, k+1}\right)$ is an eigenvector of the matrix $A_{k}^{T}$,

$$
A_{k}^{T} \vec{c}_{k}=\gamma_{0} \vec{c}_{k}
$$

where $\gamma_{0}$ is the anomalous dimension of the operator $\mathcal{O}_{n=0}$. Hence, $\gamma_{0}$ coincides with one of the eigenvalues (28), namely $\gamma_{0}=\gamma_{+}$.

Thus, we conclude that as long as the matrix $\boldsymbol{\gamma}$ retains a block-diagonal form, its eigenvalues will be degenerate. We expect that the degeneracy of the anomalous dimensions in this model will be lifted by the three-loop corrections. It is, however, simpler to consider a model where the degeneracy is absent already at the one-loop order.

\section{IV. $S U\left(N_{c}\right)$ GROSS-NEVEU-YUKAWA MODEL}

We consider the $S U\left(N_{c}\right)$ extension of the Gross-NeveuYukawa model [15]. This model describes a system of interacting fermion and scalar fields. (The bosonic model of this type was considered in Ref. [17-19].) The fermion field has two isotopic indices, $q=q^{i, I}$ that refer to the $S U\left(N_{c}\right)$ and $S U\left(N_{f}\right)$ global groups, respectively. The scalar field is in the adjoint representation of the $S U\left(N_{c}\right)$ group, $\sigma=t^{a} \sigma^{a}$, and we assume the standard normalization $\operatorname{tr} t^{a} t^{b}=\frac{1}{2} \delta^{a b}$ for the generators $t^{a}$. The renormalized action takes the form

$$
\begin{aligned}
S_{R}= & \int d^{d} x\left(Z_{1} \bar{q} \not \partial q+Z_{2} \operatorname{tr}(\partial \sigma)^{2}+M^{\epsilon} Z_{3} g \bar{q} \sigma q\right. \\
& \left.+\frac{1}{4 !} M^{2 \epsilon}\left(Z_{4} \lambda_{1}\left(\operatorname{tr} \sigma^{2}\right)^{2}+Z_{5} \lambda_{2} \operatorname{tr} \sigma^{4}\right)\right) .
\end{aligned}
$$

For $N_{c}=2,\left(\operatorname{tr} \sigma^{2}\right)^{2}=2 \operatorname{tr} \sigma^{4}$, so that one of the couplings becomes redundant and can be put to zero (we choose $\lambda_{1}=0$ ). Introducing the notations

$n_{f}=N_{f} \times \operatorname{tr}_{\gamma} \mathbb{1}, \quad u=g^{2} /(4 \pi)^{2}, \quad \bar{\lambda}_{i}=\lambda_{i} /(4 \pi)^{2}$,

one obtains the following one-loop renormalization constants

$$
Z_{1}=1-\frac{u}{2 \epsilon} C_{F} \quad Z_{2}=1-\frac{n_{f} u}{4 \epsilon}, \quad Z_{3}=1-\frac{1}{\epsilon} \frac{u}{2 N_{c}},
$$


where $C_{F}=\left(N_{c}^{2}-1\right) / 2 N_{c}$ and

$Z_{4}=1+\frac{\bar{\lambda}_{1}}{\epsilon} \frac{N_{c}^{2}+7}{24}+\frac{\bar{\lambda}_{2}}{6 \epsilon}\left[N_{c}-\frac{3}{2 N_{c}}\right]+\frac{1}{8 \epsilon} \frac{\bar{\lambda}_{2}^{2}}{\bar{\lambda}_{1}} \frac{N_{c}^{2}+3}{N_{c}^{2}}$,

$Z_{5}=1+\frac{\bar{\lambda}_{2}}{12 \epsilon}\left[N_{c}-\frac{9}{N_{c}}\right]+\frac{1}{2 \epsilon} \bar{\lambda}_{1}-\frac{6}{\epsilon} \frac{n_{f} u^{2}}{\bar{\lambda}_{2}}$.

For the index $\eta$, one gets

$$
\eta \equiv 2 \gamma_{q}=u C_{F}+O\left(\epsilon^{2}\right) .
$$

The one-loop $\beta$ functions take the form

$$
\begin{aligned}
\beta_{\bar{\lambda}_{1}}= & \bar{\lambda}_{1}\left(-2 \epsilon+n_{f} u+\bar{\lambda}_{1} \frac{N_{c}^{2}+7}{12}+\bar{\lambda}_{2} \frac{N_{c}^{2}-3}{6 N_{c}}\right) \\
& +\frac{1}{4} \bar{\lambda}_{2}^{2}\left(1+\frac{3}{N_{c}^{2}}\right), \\
\beta_{\bar{\lambda}_{2}}= & \bar{\lambda}_{2}\left(-2 \epsilon+n_{f} u+\bar{\lambda}_{2} \frac{N_{c}^{2}-9}{6 N_{c}}+\bar{\lambda}_{1}\right)-12 n_{f} u^{2}, \\
\beta_{u}= & 2 u\left(-\epsilon+u\left(\frac{n_{f}}{4}+\frac{N_{c}^{2}-3}{2 N_{c}}\right)\right),
\end{aligned}
$$

and for $N_{c}=2\left(\lambda_{1}=0\right)$,

$$
\beta_{\bar{\lambda}_{2}}=\bar{\lambda}_{2}\left(-2 \epsilon+n_{f} u+\frac{11}{24} \bar{\lambda}_{2}\right)-12 n u^{2} .
$$

For the critical $u$-coupling, one immediately gets

$$
u_{*}=4 \epsilon /\left(n_{f}+2 N_{c}-6 / N_{c}\right)+O\left(\epsilon^{2}\right) .
$$

To find the other two couplings, we assume that $n_{f} \gg N_{c}$, Then, one gets (up to $O\left(N_{c} / n_{f}\right)$ terms)

$$
\bar{\lambda}_{2}^{*}=\frac{96 \epsilon}{n_{f}}, \quad \bar{\lambda}_{1}^{*}=-\frac{1152 \epsilon}{n_{f}^{2}}\left(1+\frac{3}{N_{c}^{2}}\right) .
$$

The matrix $\omega_{i k}=\partial_{g_{i}} g_{k}$ at the critical point reads

$$
\omega=2 \epsilon\left(\mathbb{1}+O\left(N_{c} / n_{f}\right)\right) .
$$

Since all eigenvalues of $\omega$ are positive, the critical point, $\left(u_{*}, \bar{\lambda}_{1}^{*}, \bar{\lambda}_{2}^{*}\right)$, is IR stable. Note that although $\bar{\lambda}_{1}^{*}<0$, the scalar potential $V(\sigma)=\bar{\lambda}_{1}\left(\operatorname{tr} \sigma^{2}\right)^{2}+\bar{\lambda}_{2} \operatorname{tr} \sigma^{4}$ is positive since $\bar{\lambda}_{2}^{*}+N_{c} \bar{\lambda}_{1}^{*}>0$.

Numerical analysis shows that the stable critical point exists for all $N_{c}$ if $n_{f}$ is sufficiently large. For large $N_{c}$, the necessary condition boils down to $n_{f}>2 N_{c}$.

Let us study the renormalization of four-fermion operators in this model. First, we note that the operators (1) are not closed under renormalization, and one has to consider the extended set of operators

$$
\begin{aligned}
& \mathcal{O}_{n}=\frac{1}{n !}\left(\bar{q} \Gamma_{n}^{\mu_{1} \ldots \mu_{n}} q\right)\left(\bar{q} \Gamma_{\mu_{1} \ldots \mu_{n}}^{n} q\right), \\
& \hat{\mathcal{O}}_{n}=\frac{1}{n !}\left(\bar{q} \Gamma_{n}^{\mu_{1} \ldots \mu_{n}} t^{a} q\right)\left(\bar{q} t^{a} \Gamma_{\mu_{1} \ldots \mu_{n}}^{n} q\right) .
\end{aligned}
$$

Hereafter, we assume that all fields have different flavors. In order to write the anomalous dimension matrix, it is convenient to organize the operators into the following multiplets,

$$
X_{n}^{T}=\left(\mathcal{O}_{n}, \hat{\mathcal{O}}_{n+1}, \hat{\mathcal{O}}_{n+2}, \mathcal{O}_{n+3}\right)
$$

where $n=-1,1,3, \ldots$ (of course, the operator $\mathcal{O}_{n=-1}$ in $X_{-1}$ has to be omitted.).

At the critical point, the RGE for the operators $X_{n}$ can be written in the form

$$
\left(M \frac{d}{d M}+2 \eta+H_{n}\right) X_{n}=-u_{*} \frac{N_{c}^{2}-4}{2 N_{c}} Y_{n}
$$

where the matrix $H_{n}$ and vector $Y_{n}$ take the form

$$
H_{n}=2 u_{*}\left(\begin{array}{cccc}
C_{F}(2-n) & n+1 & 0 & 0 \\
\frac{C_{F}}{2 N_{c}}(4-n) & -\frac{1}{2 N_{c}}(n-1) & -\frac{N_{c}}{4}(n+2) & 0 \\
0 & -\frac{N_{c}}{4}(3-n) & \frac{1}{2 N_{c}} n & \frac{C_{F}}{2 N_{c}}(n+3) \\
0 & 0 & 2-n & C_{F}(n+1)
\end{array}\right), \quad Y_{n}=\left(\begin{array}{c}
0 \\
(4-n) \hat{\mathcal{O}}_{n} \\
(n+3) \hat{\mathcal{O}}_{n+3} \\
0
\end{array}\right)
$$

For $N_{c}=2$, the rhs of Eq. (49) vanishes and the anomalous dimension matrix acquires a block-diagonal form, with the block being equal to the matrix $H_{n}$. As could be expected from the discussion in the previous section, the eigenvalues of the block $H_{n}$ do not depend on $n$, and the anomalous dimensions take the following values:

$$
\gamma=\left\{6 u_{*}, \frac{9}{2} u_{*}, 2 u_{*},-\frac{3}{2} u_{*}\right\} .
$$

For $N_{c}>2$, Eqs. (49) do not decouple for different $n$ and, although they can be reduced to the three term recurrence relations, are still too complicated to be solved analytically. The problem becomes more tractable in the large $N_{c}$ limit. In this limit, $N_{c} \rightarrow \infty$, with $N_{c} / n_{f}$ being fixed, the operators with and without a hat decouple from each other. Moreover, there is no mixing within the operators $\mathcal{O}_{n}$ themselves so that each 
operator $\mathcal{O}_{n}$ evolves autonomously in this limit. The anomalous dimensions of the operators $\mathcal{O}_{n}$ with even $n$ and odd $n$ are

$$
\begin{aligned}
& \gamma_{n}^{+}=u_{*} N_{c}(n-1)+O\left(\epsilon^{2}\right), \\
& \gamma_{n}^{-}=u_{*} N_{c}(3-n)+O\left(\epsilon^{2}\right),
\end{aligned}
$$

respectively. At the same time, the operator $\hat{\mathcal{O}}_{n}$ satisfies the following equation:

$$
\begin{aligned}
& \left(M \partial_{M}+2 \eta\right) \hat{\mathcal{O}}_{n} \\
& =\frac{u_{*} N_{c}}{2}(-1)^{n}\left[(n+1) \hat{\mathcal{O}}_{n+1}+(n-5) \hat{\mathcal{O}}_{n-1}\right]
\end{aligned}
$$

Looking for the eigenoperator in the form

$$
\hat{\mathcal{O}}=\sum_{n}(-1)^{\frac{n(n-1)}{2}} c_{n} \hat{\mathcal{O}}_{n},
$$

one finds that, if the coefficients $c_{n}$ satisfy the recurrence relation

$$
2 \lambda c_{n}=n c_{n-1}-(n-4) c_{n+1},
$$

then $\left(M \partial_{M}+\gamma_{\lambda}\right) \hat{\mathcal{O}}_{\lambda}=0$, where $\gamma_{\lambda}=u_{*} N_{c}(1-\lambda)$.

As was discussed in Sec. II, the solutions to (53) must ensure that the correlator of eigenoperators $\left\langle\hat{\mathcal{O}}_{\lambda}(x) \hat{\mathcal{O}}_{\lambda^{\prime}}(0)\right\rangle$ is finite.

For the "physical" operators (such that not all $c_{n}=0$, for $n<5$ ), one easily obtains

$$
\begin{aligned}
\hat{\mathcal{O}}_{\lambda= \pm 2} & =\sum_{n}( \pm 1)^{n}(-1)^{\frac{1}{2} n(n-1)} \hat{\mathcal{O}}_{n}, \\
\hat{\mathcal{O}}_{\lambda= \pm 1} & =\sum_{n}( \pm 1)^{n}(n-2)(-1)^{\frac{1}{2} n(n-1)} \hat{\mathcal{O}}_{n}, \\
\hat{\mathcal{O}}_{\lambda=0} & =\sum_{n}(-1)^{\frac{1}{2} n(n-1)}(n-1)(n-3) \hat{\mathcal{O}}_{n} .
\end{aligned}
$$

All other solutions of the recurrence relation (53) have the following form,

$$
\begin{aligned}
c_{k+5}(\lambda) \equiv t_{k}(\lambda) & =\frac{1}{2 \pi i} \oint \frac{d z}{z^{k+1}}(1-z)^{-3+\lambda}(1+z)^{-3-\lambda} \\
& =(-1)^{k} \frac{(3+\lambda)_{k}}{k !}{ }_{2} F_{1}\left(\begin{array}{c}
-k, 3-\lambda \\
-k-2-\lambda
\end{array} \mid-1\right),
\end{aligned}
$$

where the integration contour encircles the point $z=0$. Since the coefficients $c_{n}=0$ for $n<5$, the corresponding eigenoperator is built from the evanescent operators only. The functions $t_{k}(\lambda)$ are polynomials of degree $k$ in $\lambda$, (anti) symmetric under $\lambda \rightarrow-\lambda, \quad t_{k}(\lambda)=(-1)^{k} t_{k}(-\lambda)$. The asymptotic of $t_{k}(\lambda)$ for large $k$ reads

$$
t_{k}(\lambda)=\frac{k^{2-\lambda}}{\Gamma(2-\lambda)}+(-1)^{k} \frac{k^{2+\lambda}}{\Gamma(2+\lambda)}+\ldots
$$

They form a complete orthonormal system,

$$
\int_{-\infty}^{\infty} d \lambda \varkappa(\lambda) t_{k}(i \lambda) \overline{t_{j}(i \lambda)}=\delta_{k j} \frac{(k+5) !}{32 k !},
$$

with respect to the measure

$$
\varkappa(\lambda)=\frac{\lambda\left(1+\lambda^{2}\right)\left(4+\lambda^{2}\right)}{\sinh \pi \lambda},
$$

which coincides, by chance, with the measure (14). It implies, in particular, that $t_{2 k}(i \lambda) \sim s_{k}(\lambda)$. We discuss it in more detail in Appendix B.

In order to fix the allowed values of $\lambda$, we consider the correlator of two eigenoperators. At the leading order it takes the form

$$
\left\langle\hat{\mathcal{O}}_{\lambda}(x) \hat{\mathcal{O}}_{\lambda^{\prime}}(0)\right\rangle \sim|x|^{-12+8 \epsilon} R\left(\lambda, \lambda^{\prime}\right),
$$

where the residue $R$ is given by the sum [see Eq. (17)]

$$
R\left(\lambda, \lambda^{\prime}\right)=\sum_{k>0}(-1)^{k} \frac{k !}{(k+5) !} t_{k}(\lambda) t_{k}\left(\lambda^{\prime}\right) .
$$

The sum diverges unless $\operatorname{Re} \lambda=0$. For imaginary $\lambda$ the correlator (59) exists in the sense of distributions. Thus the anomalous dimensions of the operator $\hat{\mathcal{O}}_{\lambda}$ is complex, $\gamma_{\lambda}=u_{*} N_{c}(1-\lambda)$.

One notices that there is a certain resemblance between the anomalous dimensions of four-fermion operators in the $S U\left(N_{c}\right) \times S U\left(N_{f}\right)$ GNY model and QED. Mixing among evanescent operators results in a continuous spectrum. In QED, the anomalous dimensions stay real, although negative, while in the GNY model they become complex. Of course, it is not excluded that this effect is an artifact of the one-loop approximation. Indeed, the spectrum is mainly determined by details of the anomalous dimension matrix at large $n$. At one loop, the matrix elements $\gamma_{n m}$ grow with $n$ as $\epsilon n^{2}$ and $\epsilon n$ in QED and in the GNY model, respectively. One has all reasons to expect that higher-order corrections will scale as $\left(\epsilon n^{2}\right)^{k}$ and $(\epsilon n)^{k}$. Whenever $\epsilon n, \epsilon n^{2} \sim O(1)$, these corrections have to be resummed. Such a resummation can drastically change the large $n$ behavior of the matrix elements. ${ }^{2}$

Finally, we consider an example to show that the construction of operators with "good" scaling properties is not always possible. Let $\mathcal{O}_{n}$ and $\mathcal{O}_{n}^{\prime}$ be the operators introduced in Sec. III, Eq. (29). These two sets of operators are related to each other by the Fierz transformation (30).

\footnotetext{
${ }^{2}$ In QED, the anomalous dimension matrix in the physical sector at two loops was obtained in [20].
} 
The correlators of the operators

$$
\begin{aligned}
f_{n m}(x)=\left\langle\mathcal{O}_{n}(x) \mathcal{O}_{m}(0)\right\rangle & =\left\langle\mathcal{O}_{n}^{\prime}(x) \mathcal{O}_{m}^{\prime}(0)\right\rangle, \\
f_{n m}^{\prime}(x) & =\left\langle\mathcal{O}_{n}(x) \mathcal{O}_{m}^{\prime}(0)\right\rangle,
\end{aligned}
$$

are well defined in the perturbative expansion (here, for definiteness, we consider QED model) and satisfy the same RGEs. Namely, for $\varphi=f, f^{\prime}$ one gets (at the critical point)

$$
M \partial_{M} \varphi_{n m}+(\gamma \varphi)_{n m}+\left(\varphi \gamma^{T}\right)_{n m}=0 .
$$

Going over to the operators $\mathcal{O}_{\nu}=\sum_{n} c_{n}(\nu) \mathcal{O}_{n}$ $\left(\mathcal{O}_{\nu}^{\prime}=\sum_{n} c_{n}(\nu) \mathcal{O}_{n}^{\prime}\right)$ one can bring the correlator $f_{n m}$ into the form (24). The coefficients $c_{n}(\nu)$ are determined by two conditions: first, they have to diagonalize the matrix $\gamma, \gamma_{n m} c_{m}(\nu) \sim c_{n}(\nu)$ and second, the product $\left(c_{n}(\nu) f_{n m}(x) c_{m}\left(\nu^{\prime}\right)\right)$ should exist in the sense of distributions.

Proceeding along the same lines with the correlator $f_{n m}^{\prime}$, one finds that while the first condition leads to the same vectors $c_{n}(\nu)$, the normalization condition changes. Now it reads (at the leading order in $\epsilon$ )

$$
R^{\prime}\left(\nu, \nu^{\prime}\right) \sim \sum_{n m} c_{n}(\nu) \Omega_{n m} \omega_{m} c_{m}\left(\nu^{\prime}\right)<\infty
$$

The matrix $C_{n m}=\Omega_{n m} \omega_{m}$ is symmetric in $n, m$ and grows as $\sim n^{m}$ for large $n$ and fixed $m$. It is easy to see that the sums in Eq. (63) diverge for any $\nu, \nu^{\prime}$. It means that while $f_{n m}^{\prime}$ correlators satisfy exactly the same RGE as $f_{n m}$, the former cannot be brought to the form (24).

This statement can also be formulated as follows. The matrix $\gamma$ commutes with the matrix $\Omega$, e.g., $\gamma \Omega=\Omega \gamma$. However, while $c_{n}(\nu)$ is an eigenvector of $\gamma$, it does not belong to the domain of the operator $\Omega$, i.e., $\sum \Omega_{n m} c_{m}(\nu)$ diverges.

The conclusion is that in noninteger dimensions, the possibility of representing the correlator $\left\langle\prod_{k} \mathcal{O}_{k}\left(x_{k}\right)\right\rangle$ as a sum of the correlators with "good" scaling properties depends on the operators $\mathcal{O}_{k}$ in question.

\section{SUMMARY}

We have considered the renormalization of four-fermion operators in the critical QED and extended GNY models. The anomalous dimension matrix in both models is of infinite size so that in order to make the diagonalization problem well defined, additional restrictions have to be imposed on the solutions. It is natural to demand for the correlation functions of the eigenoperators to be finite in the $\epsilon$ expansion, Eq. (2). By diagonalizing the anomalous dimension matrix in both models, we found that in both cases the spectrum is continuous and, for the extended GNY model, complex. Moreover, we argued that not all correlators can be expanded as a sum (integral) of contributions with specific scale dependence. We expect that all these properties are likely to be true in general for theories with fermions in $d<4$ dimensions.

It is expected that in the $d \rightarrow 3$ limit, these continuous spectrum operators should somehow decouple from the physical operators so that the evanescent operators can be consistently put to zero. Clearly, this property is hard to check within the $\epsilon$ expansion where only a few terms in the series could be calculated. It seems that alternative approaches such as the $1 / N$ expansion are better suited for this purpose.

\section{ACKNOWLEDGMENTS}

We are grateful to Michael Kelly for collaboration in the early stages of this project and V. Braun for useful comments. This work was supported by the DFG Grants No. BR 2021/7-1 (Y. J.), No. MO 1801/1-3 (A. M.) and by Russian Scientific Foundation Project No. 14-11-00598 (A. M.).

\section{APPENDIX A: $\gamma$ MATRICES IN $\boldsymbol{d}$ DIMENSIONS}

The antisymmetrized product of $\gamma$ matrices is defined as

$$
\Gamma_{\mu}^{(n)} \equiv \Gamma_{\mu_{1} \ldots \mu_{n}}^{(n)}=\frac{1}{n !} \sum_{P}(-1)^{P} \gamma_{\mu_{i_{1}}} \ldots \gamma_{\mu_{i_{n}}}
$$

where the sum is taken over all permutations. Below we collect some formulas which were helpful for the calculation. The effective technique for handling $\gamma$-matrices can be found in Refs. [21,22]. Let us denote

$$
\Gamma_{n} \otimes \Gamma_{n} \equiv \Gamma_{\mu_{1} \ldots \mu_{n}}^{(n)} \otimes \Gamma_{\mu_{1} \ldots \mu_{n}}^{(n)} .
$$

Then one finds

$$
\begin{aligned}
\gamma^{\mu} \Gamma_{n} \gamma_{\mu} \otimes \Gamma_{n}= & (-1)^{n}(d-2 n) \Gamma_{n} \otimes \Gamma_{n} \\
\gamma_{\mu} \Gamma_{n} \otimes \gamma^{\mu} \Gamma_{n}= & \Gamma_{n} \gamma_{\mu} \otimes \Gamma_{n} \gamma^{\mu}=\Gamma_{n+1} \otimes \Gamma_{n+1} \\
& +n(d-n+1) \Gamma_{n-1} \otimes \Gamma_{n-1} \\
\gamma_{\mu} \Gamma_{n} \otimes \Gamma_{n} \gamma^{\mu}= & \Gamma_{n} \gamma_{\mu} \otimes \gamma^{\mu} \Gamma_{n}=(-1)^{n}\left(\Gamma_{n+1)} \otimes \Gamma_{n+1}\right. \\
& \left.-n(d-n+1) \Gamma_{n-1} \otimes \Gamma_{n-1}\right) .
\end{aligned}
$$

The Fierz identity in $d$ dimensions has the form

$$
\Gamma_{n}^{\alpha \beta} \otimes \Gamma_{n}^{\gamma \delta}=\sum_{m=0}^{\infty} \Omega_{n k}(d) \Gamma_{m}^{\alpha \delta} \otimes \Gamma_{m}^{\gamma \beta} .
$$

The Fierz coefficients $\Omega_{n m}$ can be written as follows [22]:

$$
\begin{aligned}
\Omega_{n m}(d)= & \frac{1}{2}(-1)^{\frac{n(n-1)}{2}+\frac{m(m-1)}{2}} / \operatorname{tr} \mathbb{1} \\
& \times\left[1+(-1)^{m}+(-1)^{n}-(-1)^{n+m}\right] \\
& \times\left.\frac{1}{m !} \frac{d^{n}}{d x^{n}}(1+x)^{d-m}(1-x)^{m}\right|_{x=0} .
\end{aligned}
$$


The matrix $\Omega$ has to satisfy the consistency relation,

$$
\sum_{m=0}^{\infty} \Omega_{n m}(d) \Omega_{m k}(d)=\delta_{n k}
$$

The series converges in the region $n, k \leq d$ and for other $d$, it has to be understood as an analytical continuation [23]. With the help of the representation (A5), the sum in (A6) can be easily evaluated resulting in $\delta_{n k} 2^{d} / \operatorname{tr}_{\gamma}^{2} \rrbracket$. Thus the consistency relation (A6) holds only if the trace of the unit matrix is chosen to be [23],

$$
\operatorname{tr}_{\gamma} \mathbb{1}=2^{d / 2} .
$$

Notice that this expression coincides with the dimensions of the canonical (finite-dimensional) $\gamma$ matrix representation only for even $d$.

\section{APPENDIX B: HAHN POLYNOMIALS}

Here we collected some basic facts about the dual continuous Hahn polynomials, $S_{n}\left(x^{2}, a, b, c\right)$, which are defined as [13]

$$
S_{n}(x, a, b, c)={ }_{3} F_{2}\left(\begin{array}{c}
-n, a+i x, a-i x \\
a+b, a+c
\end{array} \mid 1\right) .
$$

They satisfy the recurrence relation

$$
\left(A_{n}+C_{n}-a^{2}-x^{2}\right) S_{n}(x)=C_{n} S_{n-1}(x)+A_{n} S_{n+1}(x),
$$

where

$$
\begin{aligned}
& C_{n}=n(n+b+c-1), \\
& A_{n}=(n+a+b)(n+a+c) .
\end{aligned}
$$

They form a complete orthonormal system on $\mathbf{L}^{2}\left(\mathbb{R}_{+}\right)$,

$$
\begin{aligned}
& \frac{1}{2 \pi} \int_{0}^{\infty} d x w(x, a, b, c) S_{n}(x) S_{m}(x) \\
& =\delta_{m n} n ! \Gamma(n+b+c) \frac{\Gamma(a+b) \Gamma(a+c)}{(a+b)_{n}(a+c)_{n}},
\end{aligned}
$$

where the measure function reads

$$
w(x, a, b, c)=\frac{|\Gamma(a+i x) \Gamma(b+i x) \Gamma(c+i x)|^{2}}{|\Gamma(2 i x)|^{2}} .
$$

Next, we demonstrate that the polynomials in Eq. (11) and (55) coincide, $s_{n}(\lambda) \sim t_{2 n}(i \lambda)$. Let us consider the recurrence relation,

$$
2 \lambda p_{n}=(n+2 \mu-1) p_{n-1}-(n+1) p_{n+1},
$$

which for $\mu=3$ is the recurrence relation for the polynomial $t_{n}(\lambda)$. The solutions have the form

$$
\begin{aligned}
p_{n}(\lambda) & =\frac{1}{2 \pi i} \oint \frac{d z}{z^{n+1}}(1-z)^{-\mu+\lambda}(1+z)^{-\mu-\lambda} \\
& =(-1)^{n} \frac{(\mu+\lambda)_{n}}{n !}{ }_{2} F_{1}\left(\begin{array}{c}
-n, \mu-\lambda \\
1-n-\mu-\lambda
\end{array} \mid-1\right) .
\end{aligned}
$$

After rescaling $t_{n}=(2 \mu)_{n} / n ! b_{n}$, the recurrence relation takes the form

$$
2 \lambda b_{n}=n b_{n-1}-(n+2 \mu) b_{n+1} .
$$

After some algebra, it can be transformed to the equation

$$
\begin{aligned}
4\left(\lambda^{2}-\mu^{2}\right) b_{n}= & (n+2 \mu)(n+2 \mu+1)\left(b_{n+2}-b_{n}\right) \\
& +n(n-1)\left(b_{n-2}-b_{n}\right),
\end{aligned}
$$

which involves the even/odd polynomials $p_{n}$ only. Having put $n=2 k(n=2 k+1)$ one find that (B7) coincides with the defining relation for the continuous dual Hahn polynomials, $S_{k}(i \lambda, \mu, 0,1 / 2)$ for even $n$, and $S_{k}(i \lambda, \mu, 1 / 2,1)$ for odd one. Taking into account the initial conditions $p_{n=0}=b_{0}=1\left(b_{1}=-\lambda / \mu\right)$, one gets

$$
\begin{aligned}
& { }_{3} F_{2}\left(\begin{array}{c}
-k, \mu+\lambda, \mu-\lambda \mid \\
\mu, \mu+\frac{1}{2}
\end{array}\right) \\
& =\frac{(\mu+\lambda)_{2 k}}{(2 \mu)_{2 k}} F_{1}\left(\begin{array}{c}
-2 k, \mu-\lambda \\
1-2 k-\mu-\lambda
\end{array} \mid-1\right)
\end{aligned}
$$

and

$$
\begin{aligned}
& \frac{\lambda}{\mu_{3}} F_{2}\left(\begin{array}{c}
-k, \mu+\lambda, \mu-\lambda \\
\mu+\frac{1}{2}, \mu+1
\end{array} \mid 1\right) \\
& =\frac{(\mu+\lambda)_{2 k+1}}{(2 \mu)_{2 k+1}} F_{1}\left(\begin{array}{c}
-2 k-1, \mu-\lambda \\
-2 k-\mu-\lambda
\end{array} \mid-1\right) .
\end{aligned}
$$

Having put $\mu=3$ in the relation (B8), one finds that $s_{k}(\lambda)=6(2 k) ! /(2 k+5) ! t_{2 k}(i \lambda)$. 
[1] K. G. Wilson and J. B. Kogut, Phys. Rep. 12, 75 (1974).

[2] D. V. Batkovich, K. G. Chetyrkin, and M. V. Kompaniets, Nucl. Phys. B906, 147 (2016).

[3] M. V. Kompaniets and E. Panzer, Phys. Rev. D 96, 036016 (2017).

[4] M. Hogervorst, S. Rychkov, and B. C. van Rees, Phys. Rev. D 93, 125025 (2016).

[5] Y. Ji and M. Kelly, Phys. Rev. D 97, 105004 (2018).

[6] A. J. Buras and P. H. Weisz, Nucl. Phys. B333, 66 (1990).

[7] M. J. Dugan and B. Grinstein, Phys. Lett. B 256, 239 (1991).

[8] S. Herrlich and U. Nierste, Nucl. Phys. B455, 39 (1995).

[9] J. A. Gracey, Nucl. Phys. B, Proc. Suppl. 51C, 24 (1996).

[10] R. D. Pisarski, Phys. Rev. D 29, 2423 (1984).

[11] T. Appelquist, D. Nash, and L. C. R. Wijewardhana, Phys. Rev. Lett. 60, 2575 (1988).

[12] A. V. Kotikov, V. I. Shilin, and S. Teber, Phys. Rev. D 94, 056009 (2016).

[13] R. Koekoek, P. A. Lesky, and R. F. Swarttouw, in Hypergeometric Orthogonal Polynomials and Their q-Analogues, edited by Tom H. Koornwinder, Springer Monographs in Mathematics (Springer-Verlag, Berlin, 2010), pp. xx+578.

[14] G. E. Andrews, R. Askey, and R. Roy, Special Functions, in Encyclopedia of Mathematics and its Applications Vol. 71 (Cambridge University Press, Cambridge, England, 1999), pp. xvi+664.

[15] J. Zinn-Justin, Nucl. Phys. B367, 105 (1991).

[16] L. N. Mihaila, N. Zerf, B. Ihrig, I. F. Herbut, and M. M. Scherer, Phys. Rev. B 96, 165133 (2017).

[17] E. Vicari and J. Zinn-Justin, New J. Phys. 8, 321 (2006).

[18] N. V. Antonov, M. V. Kompaniets, and N. M. Lebedev, J. Phys. A 46, 405002 (2013).

[19] N. V. Antonov, M. V. Kompaniets, and N. M. Lebedev, Theor. Math. Phys. 190, 204 (2017).

[20] L. Di Pietro and E. Stamou, J. High Energy Phys. 12 (2017) 054.

[21] A. D. Kennedy, J. Math. Phys. (N.Y.) 22, 1330 (1981).

[22] A. N. Vasiliev, S. E. Derkachov, and N. A. Kivel, Theor. Math. Phys. 103, 487 (1995).

[23] L. V. Avdeev, Theor. Math. Phys. 58, 203 (1984). 